\title{
Calcium-sensing receptor in nutrient sensing: an insight into the modulation of intestinal homoeostasis
}

\author{
Guangmang $\mathrm{Liu}^{1,2 *}$, Wei Cao ${ }^{1,2}$, Gang Jia ${ }^{1,2}$, Hua Zhao ${ }^{1,2}$, Xiaoling Chen ${ }^{1,2}$ and Jing Wang ${ }^{3}$ \\ ${ }^{1}$ Institute of Animal Nutrition, Sichuan Agricultural University, Chengdu 611130, Sichuan, People's Republic of China \\ ${ }^{2}$ Key Laboratory for Animal Disease-Resistance Nutrition of China Ministry of Education, Chengdu 611130, Sichuan, \\ People's Republic of China \\ ${ }^{3}$ Maize Research Institute, Sichuan Agricultural University, Chengdu 611130, Sichuan, People's Republic of China \\ (Submitted 22 January 2018 - Final revision received 23 June 2018 - Accepted 1 July 2018 - First published online 15 August 2018)
}

\section{Abstract}

The animal gut effectively prevents the entry of hazardous substances and microbes while permitting the transfer of nutrients, such as water, electrolytes, vitamins, proteins, lipids, carbohydrates, minerals and microbial metabolites, which are intimately associated with intestinal homoeostasis. The gut maintains biological functions through its nutrient-sensing receptors, including the Ca-sensing receptor (CaSR), which activates a variety of signalling pathways, depending on cellular context. CaSR coordinates food digestion and nutrient absorption, promotes cell proliferation and differentiation, regulates energy metabolism and immune response, stimulates hormone secretion, mitigates secretory diarrhoea and enhances intestinal barrier function. Thus, CaSR is crucial to the maintenance of gut homoeostasis and protection of intestinal health. In this review, we focused on the emerging roles of CaSR in the modulation of intestinal homoeostasis including related underlying mechanisms. By elucidating the relationship between CaSR and animal gut homoeostasis, effective and inexpensive methods for treating intestinal health imbalance through nutritional manipulation can be developed. This article is expected to provide experimental data of the effects of CaSR on animal or human health.

Key words: Calcium-sensing receptor: Intestinal homoeostasis: Intestinal barrier: Inflammatory responses: Secretory diarrhoea

The intestine is a crucial interface for nutrient and non-nutrient substances and energy exchange between an animal and its environment; this part of the digestive system provides the first barrier against hazardous substances, such as carcinogens, dietary-derived mutagens and oxidants ${ }^{(1)}$. The primary role of the intestine is to digest food and absorb nutrients that are subsequently transported to different tissues and organs via blood circulation for body growth. Moreover, the intestine can trigger signals to the central nervous system, thereby influencing the balance among internal environments, substances and energy. The integrated structures and normal functions of the intestine are directly related to the overall health status of the body, suggesting that maintenance of gut homoeostasis is important for animal growth and development. Intestinal homoeostasis is a state of dynamic equilibrium achieved through complex interactions between the intestinal mucosa, intestinal immune barrier, enteric microorganisms, intestinal nutrients and intestinal metabolites ${ }^{(2)}$. Gut homoeostasis plays a significant role in normal physiological functions, such as nutrient absorption, energy metabolism and reduction of intestinal infections; thus, imbalance can induce numerous diseases, including obesity, neurodevelopmental disorder and inflammatory bowel disease ${ }^{(3,4)}$.

Nutrient-sensing receptors are present in the mammalian epithelium lining the intestine and can effectively detect and respond according to the state of the microbial environment, especially microbial and nutrient composition. The receptors regulate normal intestinal functions, such as absorption and secretion, according to the state of digestion and nutrient availability. The receptors also regulate gut permeability, integrity and immunity according to the structure of the intestinal flora or the organisation of the microbial population. One of the important nutrient-sensing receptors is the extracellular Ca-sensing receptor (CaSR).

CaSR, a well-conserved and ancient guanine nucleotidebinding protein ( $G$ protein)-coupled receptor (GPCR), maintains systemic extracellular $\mathrm{Ca}^{2+}$ homoeostasis by modulating parathyroid hormone secretion ${ }^{(5,6)}$. Notably, CaSR plays other crucial roles in the physiology and pathophysiology of cellular functions involved in the growth, development and health of $\operatorname{organisms}^{(7-9)}$. CaSR can activate various tissue-specific signalling pathways upon agonist or allosteric activator stimulation

Abbreviations: CaSR, Ca-sensing receptor; CCK, cholecystokinin; ECD, extracellular domain; IP3, inositol triphosphate; NHE, sodium-hydrogen exchanger; PDE, phosphodiesterase; PLC, phospholipase C; Ror2, receptor tyrosine kinase like orphan receptor 2; TAB, TAK1-binding protein; TNFR, TNF- $\alpha$ receptor. 
and thus play critical roles in various biological processes, such as hormone secretion, cell proliferation, cell differentiation, apoptosis, ion channel activity, cell membrane potential regulation and modulation of signalling molecules for gene and protein expression $^{(5,6,10,11)}$. Abnormal CaSR expression and/or activity are closely related not only to disorders of the parathyroid gland but also to other diseases, such as diarrhoea, colitis and neoplasia ${ }^{(12-15)}$. Therefore, CaSR is important in the maintenance of animal health. Elucidating the effects of CaSR on the modulation of intestinal homoeostasis offers great theoretical and practical significance. In this review, we summarised the roles of CaSR in intestinal homoeostasis and partially offered experimental data for further studies on the effects and underlying mechanisms of CaSR.

\section{Overview of calcium-sensing receptor \\ Origin of calcium-sensing receptor}

Brown et $a l .{ }^{(16)}$ first cloned approximately 5.4 kb-long transmembrane receptor gene from a bovine parathyroid gland, and the receptor utilised $\mathrm{Ca}^{2+}$ as a physiological ligand, consequently naming the receptor CaSR in 1993. CaSR is capable of sensing minor fluctuations in extracellular $\mathrm{Ca}^{2+}$ concentration (approximately $200 \mu \mathrm{mol} / \mathrm{l}$ ) and can accurately control its concentration in serum. CaSR mainly regulates parathyroid hormone secretion and $\mathrm{Ca}^{2+}$ homoeostasis.

\section{Structural characteristics of calcium-sensing receptor}

CaSR is an exceptionally large protein that contains 1078 amino acids and has three intracellular loops and a large carboxyl terminal domain. CaSR is composed of four major parts with regard to its structure, namely the extracellular domain (ECD), a cysteine-rich domain linking the ECD to the first transmembrane helix, a seven transmembrane domain and intercellular C-terminal domain ${ }^{(17-20)}$, as shown in Fig. 1. ECD is a large domain consisting of approximately 600 amino acids with an extracellular $\mathrm{N}$ terminal domain, equipped with the characteristic topology of several GPCR - a venus flytrap structure ${ }^{(19)}$. The cysteine-rich domain linking the ECD to the transmembrane domain plays an important role in conducting signals to the seven transmembrane domain ${ }^{(19)}$. ECD comprises a hydrophilic amino terminal group $\left(-\mathrm{NH}_{2}\right)$, which enables the detection of some nutrients. The venus flytrap structure is located outside of the cell and detects several nutrients, including amino acids, peptides and polyamines ${ }^{(21)}$.

\section{Distribution of calcium-sensing receptor}

CaSR is widely distributed into the tissues of the animal or human body. CaSR is not only highly expressed in the kidney, bone tissues, thyroid, parathyroid and stomach, but also in the pancreas, marrow, breasts, pituitary gland, liver and vascular smooth muscle ${ }^{(22-25)}$. CaSR can also be found in the intestine ${ }^{(26-29)}$. CaSR is expressed in the epithelial cells of intestine and enteric nervous system ${ }^{(30-32)}$. In small intestine, CaSR is expressed in both apical and basolateral membranes of villus cells ${ }^{(33)}$. CaSR is identified in the submucosa and muscularis of the ileum and jejunum ${ }^{(30,34)}$. In the large intestine, CaSR is primarily expressed in the basal, lateral and apical membranes of epithelial cells located in the crypt and villus and in the basal endocrine cells of colonic crypt ${ }^{(35-38)}$.

\section{Roles of calcium-sensing receptor in gut homoeostasis}

The intestinal mucosa enables interactions between the internal and external environments and exhibits vital functions in digestion, absorption, secretion and activity against toxic substances. Normal digestion and absorption require intact structures of the intestinal mucosal epithelia ${ }^{(39,40)}$. Many nutrients can bind to the site of CaSR, activating signalling pathways and regulating animal growth, development and health status. CaSR can maintain gut homoeostasis by accelerating development and maturation, enhance the immune system and preserve barrier functions.

\section{Calcium-sensing receptor and intestinal development}

CaSR facilitates epithelial cell proliferation and differentiation in the intestine. The intestine is the primary site for digestion and nutrient absorption. Intestinal cells in the mucosal barrier undergo continuous turnover. Cell proliferation and differentiation occur when cells migrate from the base of the intestinal crypt to its apex, and this migration is a determinant of intestinal growth and development ${ }^{(41)}$. CaSR influences

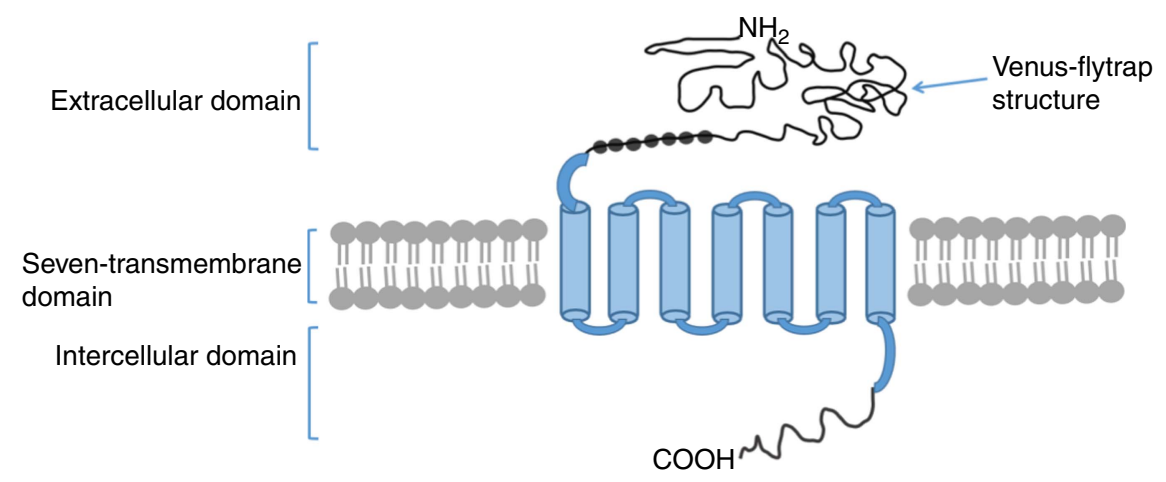

Fig. 1. Structure model of monomer of calcium-sensing receptor ${ }^{(13,14,16-18)}$. The extracellular domain contains venus flytrap structure which serves as the major ligand for agonistic interaction. Individual cysteine residues that involve the cysteine-rich domain. The carboxyl terminous is extended into the cytoplasm. 
intestinal development and growth by modulating intestinal cell proliferation and differentiation. Rey et al. ${ }^{(36)}$ first observed that epithelial CaSR deficiency in vivo results in changes in crypt structure and expansion of proliferative zones. Researchers assessed cell differentiation ex vivo by measuring $\left[{ }^{3} \mathrm{H}\right]$ thymidine incorporation into cellular DNA and found that CaSR activation causes Caco-2 cell proliferation ${ }^{(29,42)}$. CaSR expression in mice fed with a diet high in vitamin D increases colonic apoptosis ${ }^{(43)}$, suggesting a molecular link supporting positive cross-talk between the vitamin D system and CaSR. Moreover, cell apoptosis may be facilitated by CaSR and bone morphogenetic protein 2 (BMP-2), which can enhance apoptosis in colonic cell lines $^{(44)}$. In this study, the activation of CaSR by agonists increased BMP-2 gene and protein expression levels in human colonic myofibroblasts. However, CaSR knockdown by small interfering RNA or transient transfection with a dominant negative CaSR mutant suppressed BMP-2 synthesis and secretion, suggesting that CaSR promotes cell apoptosis. Apart from cell apoptosis, intestinal development and growth are intimately related to its morphological structures. In mice, poly-l-lysine feeding prevents the shortening of colon length induced by dextran sulphate sodium salt, although this effect is abrogated after the injection of a CaSR inhibitor (NPS-2143) ${ }^{(13)}$. Furthermore, spermine serves as an important CaSR agonist. Spermine supplementation effectively enhances villus height, villus width and rat jejunum surface area ${ }^{(45)}$. These observations suggest that CaSR activation by agonists promotes intestinal development.

\section{Underlying mechanisms of intestinal development induced by calcium-sensing receptor}

There is little information about signalling pathways activated by CaSR that regulate intestinal development. One study revealed the presence of CaSR on colonic epithelial cells ${ }^{(43)}$. In mice, the conditional knocking out of CaSR reduced $\beta$-catenin phosphorylation in the colon, whereas CaSR stimulation increased phosphorylation of $\beta$-catenin in a cell line derived from normal colon mucosal epithelium. Importantly, $\beta$-catenin primarily functions in the modulation of the canonical Wnt/ $\beta$-catenin signalling pathway, which is essential to intestinal development and enhances epithelial proliferation and differentiation $^{(36,46-48)}$. Thus, CaSR activation mediates gut cell proliferation and differentiation probably via $\beta$-catenin signalling and affects intestinal development and growth.

Meanwhile, CaSR regulates multitudinous downstream signalling pathways in other cells or tissues. CaSR stimulation activates G protein-mediated phospholipase C (PLC) and phosphatidylinositol bisophosphate, causing the release of inositol triphosphate (IP3) and diacylglycerol (DAG) ${ }^{(49)}$; thereafter, CaSR induces IP3-mediated intracellular $\mathrm{Ca}^{2+}$ release because IP3 can diffuse and bind to the endoplasmic reticulum while binding to its specific receptors ${ }^{(19)}$. Subsequently, an increase in intracellular DAG or $\mathrm{Ca}^{2+}$ concentration stimulates protein kinase $\mathrm{C}$, triggering mitogen-activated protein kinase (MAPK)-associated signal transduction ${ }^{(50)}$. CaSR activation also stimulates MAPK-dependent signalling proteins, such as c-Jun N-terminal kinases (JNK), P38 MAPK and extracellular

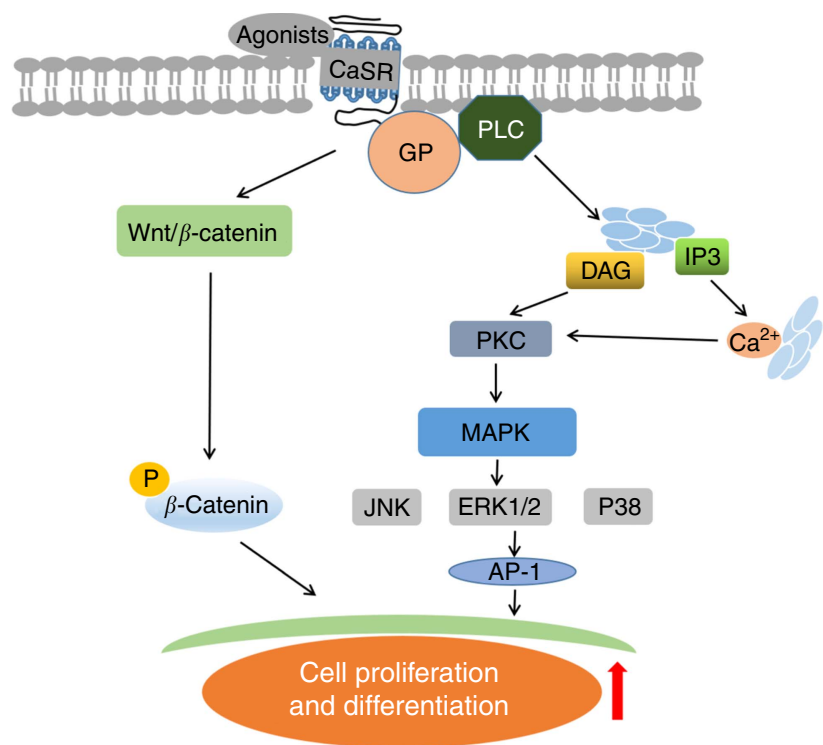

Fig. 2. Schematic diagram of underlying mechanisms of intestinal development upon calcium-sensing receptor (CaSR) activation. PLC, phospholipase C; IP3, inositol triphosphate; DAG, diacylglycerol; PKC, protein kinase C; MAPK, mitogen-activated protein kinase; JNK, c-Jun $\mathrm{N}$-terminal kinases; ERK1/2, extracellular signal-regulated kinase 1/2; GP, $\mathrm{G}$ proteins; AP-1, activator protein 1 .

signal-regulated kinase $1 / 2^{(51)}$. MAPK-dependent signal transduction strongly improves activator protein 1 (AP-1) expression. AP-1 is a regulatory factor for cell proliferation and plays vital roles in tissue development. These findings imply that CaSR participates in cell proliferation and differentiation through cascades of intracellular signalling transduction (Fig. 2).

\section{Calcium-sensing receptor and intestinal hormone secretion}

The intestine is an essential tissue for the survival and growth of mammals, owing to its primary function in digestion and nutrient absorption. Different cell types are located in the mucosa lining the intestine. These cells gradually differentiate after migrating out of the crypt, which subsequently synthesise and secrete enzymes and hormones. Intestinal hormones promote nutrient digestion and absorption, elevating nutrient availability and reducing the risk of diseases ${ }^{(52)}$ and are thus closely associated with gut homoeostasis. CaSR can modulate hormone secretion ${ }^{(53)}$. Understanding changes in hormone levels under CaSR activation and/or inhibition is fairly meaningful. Liou et al $^{(31)}$ detected the CaSR from enriched duodenal I cells that can be allosterically stimulated by a known cholecystokinin (CCK) secretagogue, L-phenylalanine. Normal mice with retained CaSR allele or double-CaSR-knockout mice exhibit distinct CCK secretion. CCK can lead to the release of digestive enzymes and bile from the pancreas and gallbladder. Native I cells originating from normal mice with retained CaSR allele supplemented with appropriate L-phenylalanine showed enhanced CCK secretion. In contrast, cells derived from doubleCaSR-knockout mice did not secrete CCK, suggesting that CaSR mediates L-amino acid-induced CCK secretion. In another experiment involving male rats, the perfused loops of the small intestine were used for the examination of CaSR-mediated gut 
peptide secretion $^{(54)}$. In this study, gluco-insulinotropic peptide (GIP), glucagon-like peptide-1 (GLP-1) and peptide tyrosine secretion from $\mathrm{K}$ and $\mathrm{L}$ cell of the small intestine were stimulated by L-amino acids, such as glutamine, phenylalanine, tryptophan, asparagine and arginine. However, these effects were suppressed by CaSR antagonist (i.e. Calhex 231) pretreatment but were induced again after the application of a specific CaSR agonist (i.e. NPS R-685). Notably, the dominating role of GLP-1 and GIP is to augment glucose-induced insulin secretion from $\beta$ cells ${ }^{(21)}$, suggesting that CaSR functions on nutrient utilisation and energy homoeostasis. As described above, CaSR expression in small intestinal epithelial cells possibly plays a significant role in sensing nutrients. Nevertheless, the detailed mechanisms of CaSR in modulating intestinal hormones secretion need further investigation.

\section{Roles of calcium-sensing receptor in intestinal barrier}

Intestinal barrier function involves the intestinal epithelium, which plays an important role in separating matters by the lumen and preventing the invasion of pathogenic substances. Under natural physiological conditions, physical, chemical, immunological and microbial barriers of the gut work together to maintain intestinal barrier function. The effects of CaSR on intestinal barrier function mainly manifest in physical, immunological and microbial barriers of the gut.

\section{Calcium-sensing receptor and intestinal physical barrier}

The epithelial monolayer of the intestine primarily serves as the physical barrier. Intestinal physical barrier refers to intact epithelium structure, which contains junctional complexes, such as tight junctions, adherens junctions, desmosomes and gap junctions ${ }^{(55)}$. Furthermore, this tissue enables the selective transport of solutes, essential nutrients and water via two routes: transcellular and paracellular pathways. The selectivity of intestinal epithelium depends on the permeability of paracellular pathway achieved through the intercellular space between adjacent cells; this permeability can be determined using fluorescein isothiocyanate (FITC) dextran ${ }^{(56)}$. Recently, an experiment using CaSR knockout mice showed increased passive transport of FITC-conjugated dextran in the colon ${ }^{(57)}$, suggesting that CaSR effectively maintains intestinal permeability by affecting the paracellular transport pathway. In addition, paracellular pathway is also associated with the state of the tight junction, which involves complex interactions between transmembrane proteins and ion transporter-associated enzymes, such as myosin lightchain kinase-1 (MLCK1). Transmembrane proteins, especially occludin and claudin, are important components of intestinal tight junctions ${ }^{(58)}$. MLCK1 is an enzyme that controls gut epithelial permeability, and its enhanced expression causes destruction of intestinal tight junctions ${ }^{(59)}$. Consistent with increased intestinal permeability, real-time PCR showed decreased claudin two mRNA level and improved MLCK1 gene expression in the colon of $\mathrm{CaSR}^{-/-}$mice ${ }^{(57)}$. Notably, the functional state of intestinal tight junctions partially relies on transepithelial resistance (TEER) ${ }^{(60)}$. $\mathrm{CaSR}^{-/-}$mice also demonstrate markedly reduced TEER and increased transepithelial conductance ${ }^{(57)}$. According to the above-mentioned results, CaSR can modulate the intestinal physical barrier.

\section{Calcium-sensing receptor and intestinal microbial barrier}

In addition to handling many dietary nutrients, the intestine harbours multiple microorganisms, especially in the colon. These microorganisms effectively prevent exogenous toxicants from entering the body. During long-term evolution process, the gut microbiota and innate intestinal structures work together to form a micro-ecosystem. Under normal conditions, the living function of an animal is maintained by a dynamic balance between the microbiota, host and environment. Consequently, the functions and health status of the intestine are closely related to its microbial composition. An increasing number of studies have illuminated the mutual relationship and bidirectional interactions between gut microbiota and nutrition through the characterisation of gut microbiota composition and function by $16 \mathrm{~S}$ ribosomal DNA (rDNA) high-throughput sequencing ${ }^{(61)}$. Many mechanisms were proposed to explain these interactions. CaSR is also expressed on immune cells and contributes to their activation; thus, there is also an interaction between the intestinal epithelial cells, the small molecules produced by the microbiome and the organismal immune cells targeted to the area. Based on these findings, we speculated that CaSR participates in the regulation of intestinal microbial barrier as a nutrient sensor by affecting microbiota composition. A previous study analysed the microbiota composition via $16 \mathrm{~S}$ rDNA-sequencing technique in steady-state $\mathrm{CaSR}^{-/-}$and wild-type mice ${ }^{(57)}$. Interestingly, no significant difference between diversity and the overall richness of the two gut microbial communities was found. Nevertheless, the results of comprehensive analyses demonstrate that epithelial CaSR deficiency alters bacterial composition. At the phylum level, an outgrowth of Deferribacteraceae was observed, which was thought to be intimately associated with inflammatory responses of the colon in Citrobacter rodentium-infected mice - a model of bacterial-mediated colitis $^{(57)}$. Knockout CaSR mice exhibit a marked decrease in beneficial lactobacilli and Clostridium ${ }^{(57)}$. Moreover, deficiency in epithelial CaSR changes the relative abundance and distribution of the Gram-positive organism Clostridium coccoides, finding its depletion in the lumen and its enrichment in the subepithelium ${ }^{(57)}$. In agreement with increased bacterial translocation and dissemination in host tissues, epithelial CaSR deficiency significantly reduces $\operatorname{Reg} 3 \beta$ and $\operatorname{Reg} 3 \gamma$ gene expressions of the distal colon ${ }^{(57)}$, which plays a vital role in encoding secreted C-type lectins that bind and protect against translocation and dissemination of Gram-negative and Grampositive bacteria ${ }^{(62,63)}$, respectively. In the light of these observations, CaSR can regulate the intestinal microbial barrier by influencing the magnitude and distribution of microbiota. However, little is known about the detailed mechanisms of this action, and further studies are thus required to address such problems.

Calcium-sensing receptor and intestinal immune barrier

Effects of calcium-sensing receptor on modulating gut immune barrier function. The mammalian intestine is not only 
the largest digestive organ but also the largest immune organ. Numerous chemical substances, including chemokines, digestive secretions, inflammatory mediators, cytokines and antimicrobial peptides, participate in intestinal barrier function. The colon exhibits a steady state of inflammation; the magnitude of which is mainly modulated by the immune barrier provided by the intestine. Therefore, we conjectured that the dysbiosis of intestinal immune barrier causes pathogenic inflammatory immune responses, and this hypothesis was verified by gene array analyses of the distal colon of wild-type and knockout CaSR mice ${ }^{(57)}$. This study revealed that $\mathrm{CaSR}^{-/-}$mice partly demonstrate the relationship between the development and maturation of intestinal immune system and CaSR. The enhanced number of B cells and expression levels of IgA were observed in the colon of $\mathrm{CaSR}^{-/-}$ mice ${ }^{57)}$. In the mesenteric lymph nodes, epithelial CaSR deficiency significantly increases the number of $\mathrm{FoxP}^{+}$regulatory $\mathrm{T}$ cells ${ }^{(57)}$ which is a subgroup of $\mathrm{CD}^{+} \mathrm{T}$ helper cell that play a central role in intestinal homoeostasis. These results suggest that CaSR locally maintains the function of intestinal immune barrier by affecting intestinal immune cells. Moreover, CaSR may be involved in the regulation of inflammatory responses closely correlated with immune status. In vitro studies reveal that epithelial CaSR inhibition increases IL- 8 secretion and IL- 6 and TNF- $\alpha$ protein expression in cultured intestinal epithelial cells ${ }^{(13,64)}$. However, epithelial CaSR activation can decrease TNF- $\alpha$ secretion in colonic myofibroblasts $^{(65)}$. Consistently, an in vivo experiment demonstrated that $\mathrm{CaSR}^{-/-}$mice showed a dramatic enhancement in the mRNA expression of numerous pro-inflammatory cytokines of the colon, such as IL-17, IL-6, IL- $1 \beta$, interferon $\gamma$ and nitric oxide synthase ${ }^{(57)}$. Glutathione analogue-induced CaSR stimulation effectively decreases inflammatory cytokine gene expression and increases anti-inflammatory cytokine gene expression, including IL-10 in mice colon ${ }^{(66)}$. Furthermore, CaSR-deficient mice exhibited more rigorous colitis induced by dextran sodium sulphate with delayed recovery relative to their littermate counterparts ${ }^{(57)}$, as evidenced by the increased weight loss, enhanced stool consistency and incomplete morphology. This result suggests that intestinal CaSR plays an important role in the alleviation of inflammatory disorders. Collectively, CaSR preserves intestinal immune barrier by participating in the modulation of intestinal immune cell maturation and inflammatory immune responses.

Underlying mechanisms of intestinal immune barrier upon calcium-sensing receptor

CaSR agonists recruit various functional signalling proteins and control diverse signalling networks, including the interference of TNF- $\alpha$-stimulated signalling pathways. As a family of such functional signalling proteins, $\beta$-arrestin family comprises multifunctional scaffold proteins capable of binding receptors phosphorylated by GPCR kinases. $\beta$-Arrestin2 is involved in CaSR-mediated anti-inflammatory effects ${ }^{(65,67)}$. In $\beta$-arrestin2deficient Caco-2 and HT-29 cells, CaSR-induced anti-inflammatory effects by GSH analogues or L-amino acid are abolished, thereby enhancing IL-8 levels. This finding implies that $\beta$-arrestin2 expression is required for CaSR-mediated antiinflammatory activity. Moreover, the interaction of $\beta$-arrestin2 with various downstream signalling components in inflammatory immune responses, such as those involving JNK and NF- $\kappa \mathrm{B}$ inhibitor protein $(\mathrm{I} \kappa \mathrm{B})$, is important ${ }^{(68,69)} . \beta$-Arrestin2 expression in intestinal epithelial cells is required for JNK and $\mathrm{I} \kappa \mathrm{B}$ phosphorylation, as demonstrated by the reduced TNF- $\alpha$ induced JNK and I $\kappa$ B phosphorylation after CaSR activation ${ }^{(66)}$. This result suggests that the underlying mechanisms of CaSRmediated anti-inflammation may involve the interaction of $\beta$-arrestin 2 with upstream signalling proteins in the TNF- $\alpha$ signalling pathway. TNF- $\alpha$ receptor (TNFR) is important in regulating inflammation. Upon the binding of TNF- $\alpha$ to TNFR, a trimeric complex is generated by interacting with transforming growth factor (TGF)- $\beta$-activated kinase 1 (TAK1), TAK1-binding protein (TAB) 1 and TAB2 in the cytoplasm when TNFR-associated factors disconnect from the receptor, mediating the activation of $\mathrm{NF}-\kappa \mathrm{B}$ and AP-1 signalling pathways and resulting in an inflammatory response $\mathrm{e}^{(70,71)}$. The studies demonstrated that GSH analogues and $\mathrm{L}$-amino acid can cause an association between $\beta$-arrestin 2 and CaSR, resulting in the interaction of $\beta$-arrestin 2 with TAB1 and decreased TNF- $\alpha$-induced TAK1-TAB1 complex generation in intestinal epithelial cells ${ }^{(64,66)}$. Similarly, CaSR activation fails to suppress TNF- $\alpha$-mediated TAB1-TAK1 association in $\beta$-arrestin2knockdown cells. These findings suggest that CaSR stimulation can maintain the intestinal immune barrier through the combination of $\beta$-arrestin 2 with TAB1 to prevent the activation of pro-inflammationdependent inflammatory signalling pathways.

In addition, the presence of $\beta$-arrestin 2 in the modulation of $\mathrm{Wnt} / \mathrm{Ca}^{2+}$ and $\mathrm{Wnt} /$ receptor tyrosine kinase like orphan receptor 2 (Ror2) pathways is essential. The activation of $\mathrm{Wnt} / \mathrm{Ca}^{2+} / \mathrm{cal}-$ modulin-dependent protein kinase II by Wnt5a accelerates NF$\kappa \mathrm{B}$ stimulation, which evokes endothelial inflammation ${ }^{(72)}$. This effect suggests that Wnt secretion and signalling play a central role in the modulation of innate and adaptive immune responses. To examine this novel role, researchers demonstrated the relationship between CaSR-mediated anti-inflammatory effects and Wnt5a/Ror2 engagement in colonic myofibroblasts and intestinal epithelial barriers challenged by lipopolysaccharide ${ }^{(65)}$. In the experiment, CaSR activation can inhibit TNF- $\alpha$ secretion in colonic myofibroblasts, enhance Ror2 expression in intestinal epithelia and induce Wnt5a secretion and expression in colonic myofibroblasts. The interaction of CaSR-mediated Wnt5a with Ror2 significantly decreased TNFR1 protein expression on intestinal epithelia. Decreased TNFR1 can reduce barrier damage by blocking TNF- $\alpha$-dependent signalling pathways. Likewise, in vivo studies showed results as described above ${ }^{(73,74)}$. In summary, these findings imply that CaSR activation inhibits TNFR1 expression on intestinal epithelia via Wnt5a/Ror2 engagement, subsequently preserving the gut immune barrier (Fig. 3).

\section{Calcium-sensing receptor and intestinal secretory diarrhoeas}

Calcium-sensing receptor mitigates intestinal secretory diarrhoeas. Both the submucosal Meissner's plexus and myenteric Auerbach's plexus cause diarrhoea when disturbed. In humans and rodents, at least $50 \%$ of the fluid secreted in cholera, rotavirus and other forms of secretory diarrhoea is caused by the activation of the enteric nervous system ${ }^{(75-78)}$. These fluids are also present in patients with irritable bowel syndrome ${ }^{(79)}$, 


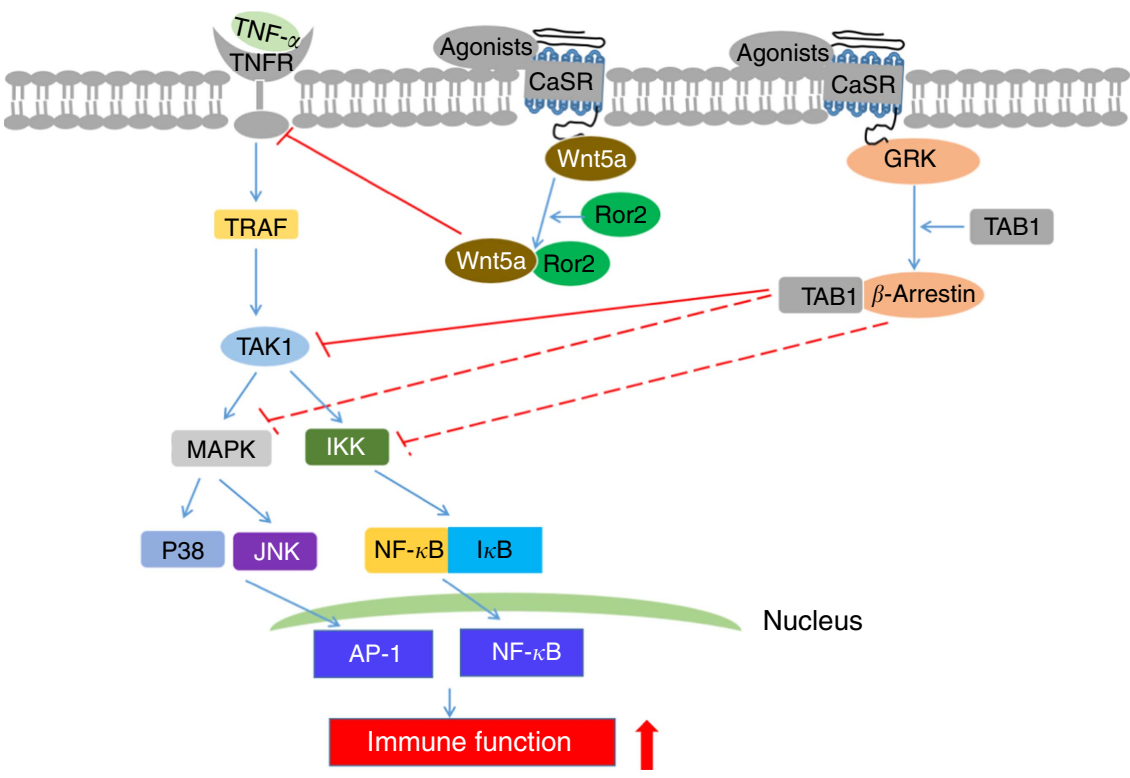

Fig. 3. Schematic diagram of underlying mechanisms of intestinal immune barrier upon calcium-sensing receptor (CaSR) activation. indicate direct and indirect inhibitory effects, respectively. TNFR, TNF- $\alpha$ receptor; TRAF, TNFR-associated factor; I $k B$, NF- $K B$ inhibitor protein; TAK1, transforming growth factor- $\beta$-activated kinase 1; GRK, G-protein-coupled cell surface receptor kinase; TAB1, TAK1 binding protein; AP-1, activator protein 1; MAPK, mitogenactivated protein kinase; IKK, IKB kinase; JNK, c-Jun N-terminal kinases; Ror2, receptor tyrosine kinase like orphan receptor 2.

inflammatory bowel disease ${ }^{(80)}$ and intestinal allergies. Colonic epithelial crypts provide a model that supports the entry of intestine-secreted fluid, and this action is mainly driven by transepithelial secretion of anions, such as $\mathrm{Cl}^{-}$. In a definitive study, the role of colonic CaSR in the regulation of intestinal fluid secretion was established through the comparison of secretagogue-stimulated $\mathrm{Cl}^{-}$responses with those of CaSR agonist $\mathrm{Ca}^{2+}$ and R568 in colonic crypts-deficient CaSR mice with wildtype controls ${ }^{(12)}$. Two effective secretagogs, namely forskolin and cholera toxin, promote basolateral membrane $\mathrm{Cl}^{-}$entry into colonocytes by detecting $N$-(ethoxycarbonylmethyl)-6-methoxyquinolinium bromide fluorescence responses sensitive to $\mathrm{Cl}^{-}$; moreover, this effect is abrogated by CaSR stimulated from either the mucosal or serosal side by $\mathrm{Ca}^{2+}$ and $\mathrm{R} 568^{(12)}$. Apart from $\mathrm{Cl}^{-}$ secretion, increased $\mathrm{HCO}_{3}^{-}$secretion caused by secretagogue also contributes to intestinal secretory diarrhoea. Thus, the suppression of $\mathrm{HCO}_{3}^{-}$transepithelial secretion by CaSR activation was investigated $^{(81)}$. Results demonstrated that R568-mediated CaSR stimulation inhibits forskolin-induced $\mathrm{HCO}_{3}^{-}$secretion in colonic apical and basolateral membranes of wild-type mice but not in $\mathrm{CaSR}^{-/-}$mice. Notably, CaSR can also be found in absorbing villus/surface cells, speculating important roles in regulating intestinal ion absorption. To address this hypothesis, the effects of CaSR activation on ion absorption in colonic crypts or mucosa derived from rats and mice are determined ${ }^{(12)}$. CaSR stimulation enhances $\mathrm{Na}^{+}$-dependent proton extrusion rate of colonocytes after increasing basolateral bath $\mathrm{Ca}^{2+}$ concentration or adding R568 in the presence of forskolin, hinting that CaSR increases $\mathrm{Na}^{+}$ absorption. Importantly, the absorption efficiency of water and some ions, such as $\mathrm{Na}^{+}$and $\mathrm{Cl}^{-}$, can be regulated by SCFA absorption, which is conducive for conserving fluid and electrolytes. The roles of CaSR in $\mathrm{Na}^{+}$absorption and $\mathrm{Cl}^{-}$secretion lead to a hypothesis that CaSR stimulation also plays a crucial role in modulating SCFA absorption. Thus, an experiment using Ussing
chamber-pH stat technique was performed ${ }^{(81)}$. As expected, R568mediated CaSR activation improves SCFA absorption in the colon of wild-type mice but not in intestinal epithelium-deficient CaSR mice. In summary, these studies reported that CaSR contributes to the amelioration of intestinal secretory diarrhoeas by blocking intestinal net fluid secretion and enhancing solute absorption.

Underlying mechanisms of reduced intestinal secretory diarrhoeas upon calcium-sensing receptor. Many factors may be involved in the underlying mechanisms of secretory diarrhoea, and cyclic nucleotide emerges as a particularly important one. Phosphodiesterase (PDE) is an enzyme that degrades cyclic nucleotides in cells ${ }^{(82)}$. CaSR-mediated increase in cyclic nucleotide degradation and decrease in forskolin-stimulated fluid secretion in the colon are maintained when intestinal cells were pretreated with isoform-nonspecific PDE inhibitor 3-isobutyl-1methylxanthine ${ }^{(12)}$. In addition, PLC can activate PDE1 activity and subsequently reverse CaSR-induced increased cyclic nucleotide degradation ${ }^{(12,82)}$. Moreover, CaSR activation induced by $\mathrm{Ca}^{2+}$ or R-568 stimulates PLC activity, which generates IP3; moreover, IP3 causes $\mathrm{Ca}^{2+}$ release in thapsigargin-sensitive stores. CaSR abrogates secretagogue-induced increase in fluid secretion by improving cyclic nucleotides destruction ${ }^{(12)}$. These observations suggest that CaSR can partly alleviate intestinal secretory diarrhoeas via PLC-PDE pathway. Fluid secretion into the lumen of colonic crypts largely depends on the role of the cystic fibrosis transmembrane conductance regulator (CFTR) chloride channels ${ }^{(83)}$. Secretagogue-induced improvements in cellular accumulation of cyclic adenosine monophosphate facilitate protein kinase A phosphorylation processes that drive the translocation of stimulated CFTR channels to the luminal plasma membrane, resulting in $\mathrm{Cl}^{-}$secretion ${ }^{(83)}$. Interestingly, the bumetanide-sensitive $\mathrm{Na}-\mathrm{K}-2 \mathrm{Cl}$ cotransporter (NKCC1) also enables $\mathrm{Cl}^{-}$entry into the lumen of colonic crypts, inducing 
continuous $\mathrm{Cl}^{-}$secretion and fluid flow ${ }^{(82)}$. NKCC1 is inhibited after CaSR activation by $\mathrm{Ca}^{2+}$ or R-568, subsequently mitigating secretory diarrhoeas ${ }^{(12)}$. Furthermore, parallel $\mathrm{Na}-\mathrm{H}$ exchanger (NHE) regulates the activities of the major components of fluid absorption. Cyclic nucleotides contribute in the suppression of $\mathrm{Na}^{+}$-dependent fluid absorption by inhibiting NHE activity ${ }^{(84)}$. Increasing basolateral bath $\mathrm{Ca}^{2+}$ concentration or adding R-568 stimulates CaSR, thereby markedly improving NHE activity up to 8 -fold ${ }^{(12)}$. This result implies that CaSR reduces secretory diarrhoeas by regulating NHE signalling and partly mitigates intestinal secretory diarrhoeas via three mechanisms, namely the PLCPDE pathway, NKCC1 signalling and NHE signalling (Fig. 4).
Calcium-sensing receptor activation and nutritional regulation. Similar to various other cell-surface receptors, CaSR can evoke a complex array of biological effects only when it combines with extracellular ligands. $\mathrm{Ca}^{2+}$ is the staple ligand for CaSR but is only one of numerous activators of this interesting receptor. Accumulating evidence proves that various nutrients, including amino acids, peptides, polyamines, glutathione analogues and alkaline polysaccharides, can be used to activate CaSR due to its unique structure, thereby implying that nutritional regulation may be developed to support CaSR-mediated maintenance of intestinal homoeostasis (Table 1).

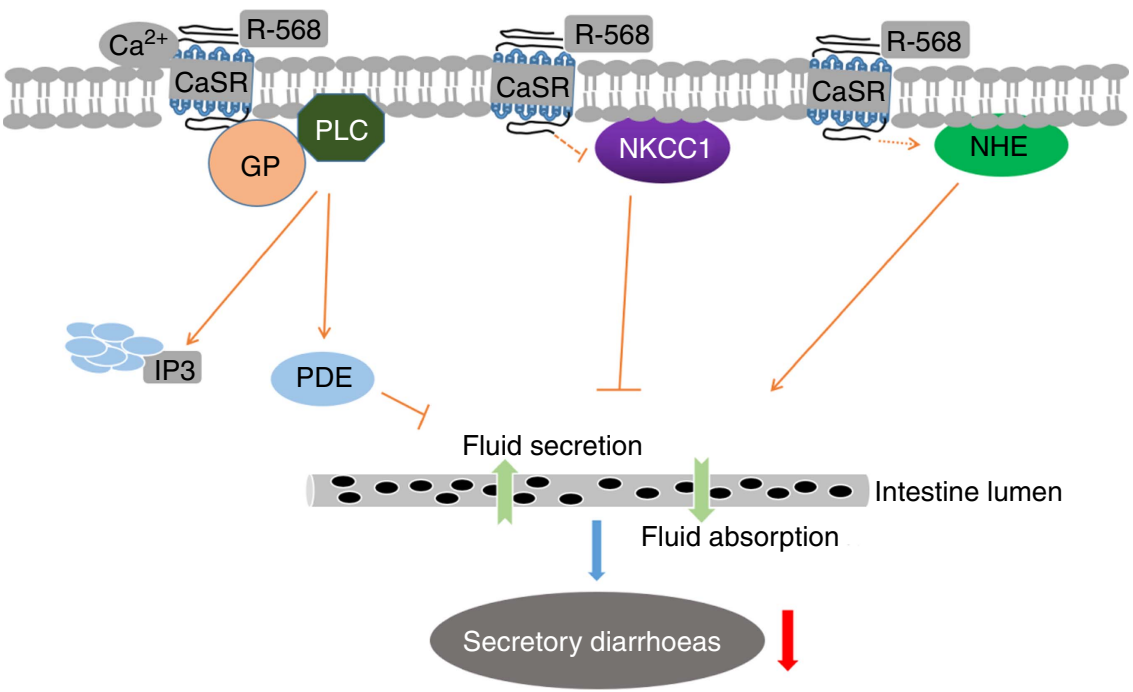

Fig. 4. Schematic diagram of underlying mechanisms of reduced intestinal secretory diarrhoeas upon calcium-sensing receptor (CaSR) activation. $-\mathrm{T}$ shapes indicate direct inhibitory effect. PLC, phospholipase C; GP, G proteins; PDE, phosphodiesterase; IP3, inositol triphosphate; NKCC1, Na-K-2Cl cotransporter; NHE, $\mathrm{Na}-\mathrm{H}$ exchanger.

Table 1. Nutritional species for calcium-sensing receptor activation regulation

\begin{tabular}{|c|c|c|c|}
\hline Nutrient & Type & Binding region & References \\
\hline \multicolumn{4}{|l|}{ L-Amino acids } \\
\hline Phe & Orthosteric agonists & Extracellular domain & $(85-90)$ \\
\hline Trp & & & \\
\hline Tyr & & & \\
\hline His & & & \\
\hline \multicolumn{4}{|l|}{ Polypeptides } \\
\hline Poly-L-arginine & Allosteric modulators & Extracellular domain & $(91-94)$ \\
\hline Poly-L-lysine & & & \\
\hline Protamine & & & \\
\hline Amyloid & & & \\
\hline$\beta$-Peptides & & & \\
\hline \multicolumn{4}{|l|}{ Polyamines } \\
\hline Spermine & Orthosteric agonists & Extracellular domain & (95) \\
\hline Spermidine & & & \\
\hline Putrescine & & & \\
\hline \multicolumn{4}{|l|}{ Glutathione analogues } \\
\hline Glutathione & Allosteric modulators & Extracellular domain & $(86,96,97)$ \\
\hline S-Methylglutathione & & & \\
\hline S-Propylglutathione & & & \\
\hline$\gamma$-Glu-Ala & & & \\
\hline \multirow{2}{*}{\multicolumn{4}{|c|}{ Alkaline polysaccharide }} \\
\hline & & & \\
\hline Chitosan oligosaccharide & Orthosteric agonists & Extracellular domain & (98) \\
\hline
\end{tabular}




\section{Conclusion and future perspectives}

Intestinal epithelium CaSR participates in the maintenance of intestinal homoeostasis, as evidenced by increased intestinal cell proliferation and differentiation, increased cell apoptosis, changed intestinal morphology, improved hormone secretion, recovered physical barrier, enhanced immune barrier, reinforced microbial compositions and alleviated secretory diarrhoeas, using in vitro, ex vivo or in vivo models. Furthermore, evidence supports the underlying mechanisms of CaSR which involve intestinal cell proliferation and differentiation, immune homoeostasis and secretory diarrhoeas. However, many questions associated with intestinal homoeostasis remain unsolved and require further studies. For example, whether CaSR improves the length, weight and digestive enzymes activities of the intestine for the acceleration of intestinal development, and whether it affects intestinal antioxidant status and metabolism for intestinal homoeostasis remain unknown. The role of CaSR in intestinal damage and repair, and the specific molecular mechanism of CaSR in the maintenance of intestinal physical and microbial barriers are unresolved. CaSR was identified as a novel and promising therapeutic target for the treatment of various gut diseases such as inflammatory bowel disease. A fundamental challenge for future studies is the exploration of the roles of CaSR in the intestine and the exact mechanisms of CaSR-mediated intestinal health from the view of nutriology. This research may provide primary experimental data of the supporting role of CaSR in the resistance of animals against diseases.

\section{Acknowledgements}

The present study was financially supported by the Program for Discipline Construction in Sichuan Agricultural University (no. 03570126).

The authors' contributions were as follows: G. L. and W. C. contributed to the preparation of the manuscript, figures and editing of the manuscript. G. J., H. Z., X. C. and J. W. were involved in the preparation of the manuscript.

The authors declare that there are no conflicts of interest.

\section{References}

1. Cencic A \& Langerholc T (2010) Functional cell models of the gut and their applications in food microbiology-a review. Int J Food Microbiol 141, S4-S14.

2. Maloy KJ \& Powrie F (2011) Intestinal homeostasis and its breakdown in inflammatory bowel disease. Nature $\mathbf{4 7 4}$, 298-306.

3. Wei X, Yang Z, Rey FE, et al. (2012) Fatty acid synthase modulates intestinal barrier function through palmitoylation of mucin 2. Cell Host Microbe 11, 140-152.

4. Hsiao EY, McBride SW, Hsien S, et al. (2013) Microbiota modulate behavioral and physiological abnormalities associated with neurodevelopmental disorders. Cell 155, 1451-1463.

5. Lin KI, Chattopadhyay N, Bai M, et al. (1998) Elevated extracellular calcium can prevent apoptosis via the calcium-sensing receptor. Biochem Biophys Res Commun 249, 325-331.

6. Ba J \& Friedman PA (2004) Calcium-sensing receptor regulation of renal mineral ion transport. Cell Calcium 35, 229-237.
7. Ward BK, Magno AL, Walsh JP, et al. (2012) The role of the calcium-sensing receptor in human disease. Clin Biochem $\mathbf{4 5}$, 943-953.

8. Tfelt-Hansen J \& Brown E (2005) The calcium-sensing receptor in normal physiology and pathophysiology: a review. Crit Rev Clin Lab Sci $\mathbf{4 2}, 35-70$.

9. Lopez-Fernandez I, Schepelmann M, Brennan SC, et al. (2015) The calcium-sensing receptor: one of a kind. Exp Physiol 100, 1392-1399.

10. Tennakoon S, Aggarwal A \& Kállay E (2016) The calciumsensing receptor and the hallmarks of cancer. Biochim Biophys Acta 1863, 1398-1407.

11. Brown EM \& MacLeod RJ (2001) Extracellular calcium sensing and extracellular calcium signaling. Physiol Rev 81, 239-297.

12. Geibel J, Sritharan K, Geibel R, et al. (2006) Calcium-sensing receptor abrogates secretagogue-induced increases in intestinal net fluid secretion by enhancing cyclic nucleotide destruction. Proc Natl Acad Sci US A 103, 9390-9397.

13. Mine $\mathrm{Y} \&$ \& Zhang $\mathrm{H}$ (2015) Anti-inflammatory effects of poly-L-lysine in intestinal mucosal system mediated by calcium-sensing receptor activation. I Agric Food Chem $\mathbf{6 3}$, 10437-10447.

14. Brennan SC, Thiem U, Roth S, et al. (2013) Calcium sensing receptor signalling in physiology and cancer. Biochim Biophys Acta 1833, 1732-1744.

15. Fraebel J, Gonzalez-Peralta R, Maximos M, et al. (2018) Extracellular calcium dictates onset, severity, and recovery of diarrhea in a child with immune-mediated enteropathy. Front Pediatr 6, 7 .

16. Brown EM, Gambag G, Riccardi D, et al. (1993) Cloning and characterization of an extracellular $\mathrm{Ca}(2+)$-sensing receptor from bovine parathyroid. Nature 366, 575-580.

17. Garrett JE, Capuano IV, Hammerland LG, et al. (1995) Molecular cloning and functional expression of human parathyroid calcium receptor cDNAs. J Biol Chem 270, 12919-12925.

18. Zhang C, Miller CL, Brown EM, et al. (2015) The calcium sensing receptor: from calcium sensing to signaling. Sci China Life Sci 58, 14-27.

19. Hofer AM \& Brown EM (2003) Extracellular calcium sensing and signalling. Nat Rev Mol Cell Biol 4, 530-538.

20. Bai M, Quinn S, Trivedi S, et al. (1996) Expression and characterization of inactivating and activating mutations in the human $\mathrm{Ca}^{2+}$-sensing receptor. J Biol Chem 271, 19537-19545.

21. Tang L, Cheng CY, Sun X, et al. (2016) The extracellular calcium-sensing receptor in the intestine: evidence for regulation of colonic absorption, secretion, motility, and immunity. Front Physiol 7, 245-262.

22. Wonneberger K, Scofield MA \& Wangemann P (2000) Evidence for a calcium-sensing receptor in the vascular smooth muscle cells of the spiral modiolar artery. J Membr Biol 175, 203-212.

23. Cheng I, Qureshi I, Chattopadhyay N, et al. (1999) Expression of an extracellular calcium-sensing receptor in rat stomach. Gastroenterology 116, 118-126.

24. Cheng I, Klingensmith ME, Chattopadhyay N, et al. (1998) Identification and localization of the extracellular calciumsensing receptor in human breast. J Clin Endocrinol Metab 83, 703-707.

25. Riccardi D, Park J, Lee WS, et al. (1995) Cloning and functional expression of a rat kidney extracellular calcium/ polyvalent cationsensing receptor. Proc Natl Acad Sci US A 92, 131-135.

26. Geibel JP \& Hebert SC (2009) The functions and roles of the extracellular $\mathrm{Ca}^{2+}$-sensing receptor along the gastrointestinal tract. Annu Rev Physiol 71, 205-217. 
27. Cheng SX, Okuda M, Hall AE, et al. (2002) Expression of calcium-sensing receptor in rat colonic epithelium: evidence for modulation of fluid secretion. Am J Physiol Gastrointest Liver Physiol 283, G240-G250.

28. Cheng SX, Geibel JP \& Hebert SC (2004) Extracellular polyamines regulate fluid secretion in rat colonic crypts via the extracellular calcium-sensing receptor. Gastroenterology 126, $148-158$.

29. Aggarwal A, Prinz-Wohlgenannt $\mathrm{M}$, Tennakoon $\mathrm{S}$, et al. (2015) The calcium-sensing receptor: a promising target for prevention of colorectal cancer. Biochim Biophys Acta 1853, 2158-2167.

30. Chattopadhyay N, Cheng I, Rogers K, et al. (1998) Identification and localization of extracellular $\mathrm{Ca}^{2+}$-sensing receptor in rat intestine. Am J Physiol 274, G122-G130.

31. Liou AP, Sei Y, Zhao X, et al. (2011) The extracellular calciumsensing receptor is required for cholecystokinin secretion in response to L-phenylalanine in acutely isolated intestinal I cells. Am J Physiol Gastrointest Liver Physiol 300, G538-G546.

32. Cheng SX (2012) Calcium-sensing receptor inhibits secretagogue-induced electrolyte secretion by intestine via the enteric nervous system. Am J Physiol Gastrointest Liver Physiol 303, G60-G70

33. Chattopadhyay N, Cheng I, Rogers K, et al. (1998) Identification and localization of extracellular $\mathrm{Ca}^{2+}$-sensing receptor in rat intestine. Am J Physiol Gastrointest Liver Physiol 274, G122-G130.

34. Gama L, Baxendale-Cox LM \& Breitwieser GE (1997) $\mathrm{Ca}^{2+}$ -sensing receptors in intestinal epithelium. Am J Physiol 273, C1168-C1175.

35. Hebert SC, Cheng S \& Geibel J (2004) Functions and roles of the extracellular $\mathrm{Ca}^{2+}$-sensing receptor in the gastrointestinal tract. Cell Calcium 35, 239-247.

36. Rey O, Chang W, Bikiel D, et al. (2012) Negative crosstalk between calcium-sensing receptor and $\beta$-catenin signaling systems in colonic epithelium. J Biol Chem 287, 1158-1167.

37. Sheinin Y, Kllay E, Wbaf F, et al. (2000) Immunocytochemical localization of the extracellular calcium sensing receptor in normal and malignant human large intestinal mucosa. $J$ Histochem Cytochem 48, 595-601.

38. Fetahu IS, Höbaus J, Aggarwal A, et al. (2014) Calcium-sensing receptor silencing in colorectal cancer is associated with promoter hypermethylation and loss of acetylation on histone 3. Int J Cancer 135, 2014-2023.

39. Flint HJ, Scott KP, Louis P, et al. (2012) The role of the gut microbiota in nutrition and health. Nat Rev Gastroenterol Hepatol 9, 577-589.

40. Peterson LW \& Artis D (2014) Intestinal epithelial cells: regulators of barrier function and immune homeostasis. Nat Rev Immunol 14, 141-153.

41. Cummins AG \& Thompson FM (2002) Effect of breast milk and weaning on epithelial growth of the small intestine in humans. Gut 51, 748-754.

42. Kállay E, Kifor O, Chattopadhyay N, et al. (1997) Calcium-dependent c-myc proto-oncogene expression and proliferation of Caco-2 cells: a role for a luminal extracellular calcium-sensing receptor. Biochem Biophys Res Commun 232, 80-83.

43. Aggarwal A, Höbaus J, Tennakoon S, et al. (2016) Active vitamin D potentiates the anti-neoplastic effects of calcium in the colon: a cross talk through the calcium-sensing receptor. J Steroid Biochem Mol Biol 155, 231-238.

44. Peiris D, Pacheco I, Spencer C, et al. (2007) The extracellular calcium-sensing receptor reciprocally regulates the secretion of BMP-2 and the BMP antagonist Noggin in colonic myofibroblasts. Am J Physiol Gastrointest Liver Physiol 292, G753-G766.

45. Cao W, Liu G, Fang T, et al. (2015) Effects of spermine on the morphology, digestive enzyme activities, and antioxidant status of jejunum in suckling rats. RSC Adv 5, 76607-76614.

46. Clevers H (2006) Wnt/ $\beta$-catenin signaling in development and disease. Cell 127, 469-480.

47. McDonald SA, Preston SL, Lovell MJ, et al. (2006) Mechanisms of disease: from stem cells to colorectal cancer. Nat Clin Pract Gastroenterol Hepatol 3, 267-274.

48. Aggarwal A, Prinz-Wohlgenannt M, Gröscel C, et al. (2014) The calcium-sensing receptor suppresses epithelial-to-mesenchymal transition and stem cell-like phenotype in the colon. Mol Cancer 14, 61.

49. Buchan AM, Squires PE, Ring M, et al. (2001) Mechanism of action of the calcium-sensing receptor in human antral gastrin cells. Gastroenterology 120, 1128-1139.

50. Schönwasser DC, Marais RM, Marshall CJ, et al. (1998) Signal-regulated kinase pathway by conventional, novel, and atypical protein kinase C isotypes. Mol Cell Biol 18, 790-798.

51. Tfelt-Hansen J, MacLeod RJ, Chattopadhyay N, et al. (2003) Calcium-sensing receptor stimulates PTHrP release by pathways dependent on PKC, p38 MAPK, JNK, and ERK1/2 in H-500 cells. Am J Physiol Endocrinol Metab 285, E329-E337.

52. Lund A, Vilsboll T, Bagger JI, et al. (2011) The separate and combined impact of the intestinal hormones, GIP, GLP-1, and GLP-2, on glucagon secretion in type 2 diabetes. Am J Physiol Endocrinol Metab 300, E1038-E1046.

53. Macleod RJ (2013) CaSR function in the intestine: hormone secretion, electrolyte absorption and secretion, paracrine noncanonical Wnt signaling and colonic crypt cell proliferation. Best Pract Res Clin Endocrinol Metab 27, 385-402.

54. Mace OJ, Schindler M \& Patel S (2012) The regulation of Kand L-cell activity by GLUT2 and the calcium-sensing receptor CaSR in rat small intestine. J Physiol 590, 2917-2936.

55. Owen JL, Cheng SX, Ge Y, et al. (2016) The role of the calcium-sensing receptor in gastrointestinal inflammation. Semin Cell Dev Biol 49, 44-51.

56. Stenman LK, Holma R, Gylling H, et al. (2013) Genetically obese mice do not show increased gut permeability or faecal bile acid hydrophobicity. Br J Nutr 110, 1157-1164.

57. Cheng SX, Lightfoot YL, Yang T, et al. (2014) Epithelial CaSR deficiency alters intestinal integrity and promotes proinflammatory immune responses. FEBS Lett 588, 4158-4166.

58. Tsukita S \& Furuse M (1999) Occludin and claudins in tightjunction strands: leading or supporting players? Trends Cell Biol 9, 268-273

59. Graham WV, Wang F, Clayburgh DR, et al. (2006) Tumor necrosis factor-induced long myosin light chain kinase transcription is regulated by differentiation-dependent signaling events. Characterization of the human long myosin light chain kinase promoter. J Biol Chem 281, 26205-26215.

60. Schmitz H, Fromm M, Bentzel CJ, et al. (1999) Tumor necrosis factor-alpha (TNFalpha) regulates the epithelial barrier in the human intestinal cell line HT-29/B6. J Cell Sci 112, 137-146.

61. Maukonen J \& Saarela M (2015) Human gut microbiota: does diet matter? Proc Nutr Soc 74, 23-36.

62. van Ampting MT, Loonen LM, Schonewille AJ, et al. (2012) Intestinally secreted C-type lectin Reg3b attenuates salmonellosis but not listeriosis in mice. Infect Immun $\mathbf{8 0}$, $1115-1120$

63. Cash HL, Whitham CV, Behrendt CL, et al. (2006) Symbiotic bacteria direct expression of an intestinal bactericidal lectin. Science 313, 1126-1130. 
64. Mine Y \& Zhang H (2015) Calcium-sensing receptor (CaSR)mediated anti-inflammatory effects of $\mathrm{L}$-amino acids in intestinal epithelial cells. J Agric Food Chem 63, 9987-9995.

65. Kelly JC, Lungchukiet P \& MacLeod RJ (2011) Extracellular calcium-sensing receptor inhibition of intestinal epithelial TNF signaling requires CaSR-mediated Wnt5a/Ror2 interaction. Front Physiol 2, 17.

66. Zhang H, Kovacs-Nolan J, Kodera T, et al. (2015) $\gamma$-Glutamyl cysteine and $\gamma$-glutamyl valine inhibit TNF- $\alpha$ signaling in intestinal epithelial cells and reduce inflammation in a mouse model of colitis via allosteric activation of the calcium-sensing receptor. Biochim Biophy Acta 1852, 792-804.

67. Gao H, Sun Y, Wu Y, et al. (2004) Identification of betaarrestin2 as a $\mathrm{G}$ protein-coupled receptor-stimulated regulator of NF-кB pathways. Mol Cell 14, 303-317.

68. DeWire SM, Ahn S, Lefkowitz RJ, et al. (2007) $\beta$-Arrestins and cell signaling. Annu Rev Physiol 69, 483-510.

69. Witherow DS, Garrison TR, Miller WE, et al. (2004) $\beta$-Arrestin inhibits NF- $\kappa$ B activity by means of its interaction with the NF-

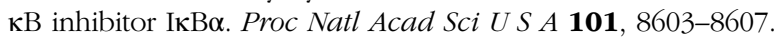

70. Liu Q, Busby JG \& Molkentin JD (2009) Novel interaction between TAK1-TAB1-TAB2 and RCAN1-calcineurin defines a signalling nodal control point. Nat Cell Biol 11, 154-161.

71. Ninomiya-Tsuji J, Kishimoto K, Hiyama A, et al. (1999) The kinase TAK1 can activate the NIK-IкB as well as the MAP kinase cascade in the IL-1 signalling pathway. Nature 398, 252-256.

72. Kim J, Kim J, Kim DW, et al. (2010) Wnt5a induces endothelial inflammation via $\beta$-catenin-independent signaling. J Immunol 185, 1274-1282.

73. Turki R, Kelly JE, Pacheco I, et al. (2012) Effect of increased dietary calcium on murine colonic CaSR expression and the effect of induced colitis on "rescued" CaSR-/PTH- (C-/P-) knockout mice. Bone 51, S23-S24.

74. MacLeod RJ (2013) Extracellular calcium-sensing receptor/ PTH knockout mice colons have increased Wnt/ $\beta$-catenin signaling, reduced non-canonical Wnt signaling, and increased susceptibility to azoxymethane-induced aberrant crypt foci. Lab Invest 93, 520-527.

75. Burleigh DE \& Borman RA (1997) Evidence for a nonneural electrogenic effect of cholera toxin on human isolated ileal mucosa. Dig Dis Sci 42, 1964-1968.

76. Field M (2003) Intestinal ion transport and the pathophysiology of diarrhea. J Clin Invest 111, 931-943.

77. Lorrot M \& Vasseur M (2007) How do the rotavirus NSP4 and bacterial enterotoxins lead differently to diarrhea? Virol J 4, 31.

78. Lundgren O, Peregrin AT, Persson K, et al. (2000) Role of the enteric nervous system in the fluid and electrolyte secretion of rotavirus diarrhea. Science 287, 491-495.

79. Wood JD (2006) Histamine, mast cells, and the enteric nervous system in the irritable bowel syndrome, enteritis, and food allergies. Gut 55, 445-447.

80. Margolis KG, Stevanovic K, Karamooz N, et al. (2011) Enteric neuronal density contributes to the severity of intestinal inflammation. Gastroenterology 141, 588-598.

81. Tang L, Peng M, Liu L, et al. (2015) Calcium-sensing receptor stimulates $\mathrm{Cl}^{-}$-and SCFA-dependent but inhibits CAMPdependent $\mathrm{HCO}_{3}^{-}$secretion in colon. Am J Physiol Gastrointest Liver Physiol 308, G874-G883.
82. Beavo JA (1995) Cyclic nucleotide phosphodiesterases: functional implications of multiple isoforms. Physiol Rev 75, 725-748.

83. Kunzelmann K \& Mall M (2002) Electrolyte transport in the mammalian colon: mechanisms and implications for disease. Physiol Rev 82, 245-289.

84. Yun CHC, Oh S, Zizak M, et al. (1997) cAMP-mediated inhibition of the epithelial brush border $\mathrm{Na}^{+} / \mathrm{H}^{+}$exchanger, NHE3, requires an associated regulatory protein. Proc Natl Acad Sci US A 94, 3010-3015.

85. Ohsu T, Amino Y, Nagasaki H, et al. (2010) Involvement of the calcium-sensing receptor in human taste perception. J Biol Chem 285, 1016-1022.

86. Roadhead GK, Mun HC, Avnali VA, et al. (2011) Allosteric modulation of the calcium-sensing receptor by $\gamma$-glutamyl peptides: inhibition of PTH secretion, suppression of intracellular cAMP levels, and a common mechanism of action with L-amino acids. J Biol Chem 286, 8786-8797.

87. Alamshah A, Spreckley E, Norton M, et al. (2017) L-Phenylalanine modulates gut hormone release and glucose tolerance, and suppresses food intake through the calcium-sensing receptor in rodents. Int J Obes $\mathbf{4 1}, 1693-1701$.

88. Conigrave AD, Quinn SJ \& Brown EM (2000) L-Amino acid sensing by the extracellular $\mathrm{Ca}^{2+}$-sensing receptor. Proc Natl Acad Sci U S A 97, 4814-4819.

89. Conigrave AD, Mun HC \& Brennan SC (2007) Physiological significance of L-amino acid sensing by extracellular $\mathrm{Ca}^{2+}$ -sensing receptors. Biochem Soc Trans 35, 1195-1198.

90. Conigrave AD \& Hampson DR (2010) Broad-spectrum amino acid-sensing class C G-protein coupled receptors: molecular mechanisms, physiological significance and options for drug development. Pharmacol Ther 127, 252-260.

91. Popivanova BK, Kitamura K, Wu Y, et al. (2008) Blocking TNF-alpha in mice reduces colorectal carcinogenesis associated with chronic colitis. J Clin Invest 118, 560-570.

92. Brown EM, Katz C, Butters R, et al. (1991) Polyarginine, polylysine, and protamine mimic the effects of high extracellular calcium concentrations on dispersed bovine parathyroid cells. J Bone Miner Res 6, 1217-1226.

93. Ye C, Ho-Pao CL, Kanazirska M, et al. (1997) Amyloid- $\beta$ proteins activate $\mathrm{Ca}^{2+}$-permeable channels through calciumsensing receptors. J Neurosci Res 47, 547-554.

94. Stix B \& Reiser G (1998) Beta-amyloid peptide 25-35 regulates basal and hormone-stimulated $\mathrm{Ca}^{2+}$ levels in cultures rat astrocytes. Neurosci Lett 243, 121-124.

95. Quinn SJ, Ye CP, Diaz R, et al. (1997) The $\mathrm{Ca}^{2+}$-sensing receptor: a target for polyamines. Am J Physiol 273, C1315-C1323.

96. Wang M, Yao Y, Kuang D, et al. (2006) Activation of family C G-protein-coupled receptors by the tripeptide glutathione. J Biol Chem 281, 8864-8870.

97. Broadhead GK, Mun HC, Avlani VA, et al. (2011) Allosteric modulation of the calcium-sensing receptor by gammaglutamyl peptides: inhibition of PTH secretion, suppression of intracellular cAMP levels, and a common mechanism of action with L-amino acids. J Biol Chem 286, 8786-8797.

98. Huang B, Xiao D, Tan B, et al. (2015) Chitosan oligosaccharide reduces intestinal inflammation that involves calcium-sensing receptor (CaSR) activation in lipopolysaccharide (LPS)-challenged piglets. J Agric Food Chem 64, 245-252. 